\title{
Anatomical Extent of Venous Reflux
}

Fedor Lurie

Received: April 28, 2020 / Published online: June 2, 2020

(c) The Author(s) 2020

\section{ABSTRACT}

Despite the recognition of the importance of the anatomical extent of venous reflux, its description in medical literature is inconsistent in terms and classifications. Recent international consensus documents provide clear definitions for the three main classes of reflux: segmental, multi-segmental, and axial. This report addresses the most important aspects of the standard definitions of the anatomical extent of venous reflux, the differences between the three classes of reflux, and the challenges of implementing this classification in clinical practice.

Keywords: Chronic venous disease; Venous insufficiency; Venous reflux

Digital features: To view digital features for this article go to https://doi.org/10.6084/m9.figshare.12377204.

F. Lurie $(\bowtie)$

Department of Vascular Surgery, Promedica Jobst

Vascular Institute, Toledo, $\mathrm{OH}$, USA

e-mail: Fedor.lurie@promedica.org

F. Lurie

Division of Vascular Surgery, University of Michigan

at Ann Arbor, Ann Arbor, MI, USA

\section{Key Summary Points}

Anatomical extent of venous reflux has been defined as segmental, multisegmental, and axial.

Axial reflux is defined as "uninterrupted retrograde venous floor from the groin to the calf."

Segmental reflux may occur in the veins with competent valves.

Axial reflux always involves incompetent valves.

Knowledge of the anatomical extent of venous reflux is important for several reasons. Understanding the relationship of refluxing veins to the site and severity of clinical manifestation may be the most important, for this knowledge serves as a basis for selecting appropriate treatment. For example, reflux in a short segment of the small saphenous vein (SSV) in the upper calf is unlikely to be related to a venous ulcer in the medial ankle. To make such a conclusion, however, requires knowledge of the existing connections between this segment and incompetent veins in the ulcer bed. Another example may be varicose veins causing symptoms in the lateral thigh and calf that 
more likely relate to reflux in the accessory saphenous vein than to an incompetent proximal segment of the great saphenous vein (GSV).

The connection between the anatomical extent of reflux and the progression of primary chronic venous disease has been consistently demonstrated by many studies [1-4]. Axial reflux and multi-segmental reflux are more prevalent in patients with chronic venous insufficiency (CVI) than in those with less advanced disease. Retrospective and prospective cohort studies have shown a consistent pattern of reflux progression from segmental to multisegmental and axial. This trend is the same regardless of the "descending" or "ascending" direction.

Despite its importance, descriptions of the anatomical extent of venous reflux in the literature are limited. The " $\mathrm{A}$ " in the abbreviated CEAP (Clinical-Etiology-Anatomy-Pathophysiology) classification describes the main groups, such as deep and superficial reflux, which are too broad to understand which segments are involved. The complete CEAP describes all individual segments but without information regarding which of these segments may be connected. Multiple attempts to create a more detailed classification have resulted in so many classes of reflux that it becomes completely impractical to follow [3, 4].

The lack of a uniform terminology to be consistently used in research and clinical publications has been one of the major barriers to progress in understanding venous disorders. Addressing this problem, an international consensus group has published The Vein Glossary, which integrates previous consensus documents and definitions commonly used in vascular literature. Universally accepted and translated to all major languages, this document defines axial reflux as "uninterrupted retrograde venous flow from the groin to the calf." Superficial reflux is defined as confined to the superficial system, deep reflux as confined to the deep system, and combined reflux as any combination of the venous systems, as long as it is an uninterrupted connection from the thigh to the groin. The process of acceptance and use of available uniform terminology is slow. It is not infrequent that the title of a paper might contain the term "axial reflux," but the paper itself does not describe any anatomical extent of the reflux, or the anatomical extent is defined differently from the definitions recommended by The Vein Glossary.

The major challenge in standardizing definitions of the anatomical extent of reflux, however, is that the current practice of identifying reflux is based on duplex ultrasound. The ultrasound scan interrogates each venous segment separately, and the anatomical extent of venous reflux is always a reconstruction of segmental information. Technologists' education, experience, and familiarity with definitions are some of the many factors leading to the variability in reflux descriptions. In addition to being an operator- and protocol-dependent test, an ultrasound scan provides information only regarding the hemodynamic phenomenon of a reversed flow (reflux), which should not be confused with valvular incompetence. Reversed flow (reflux) can occur in a segment of competent saphenous vein. For example, a GSV segment between two competent valves can have a reversed flow from a proximal tributary into a

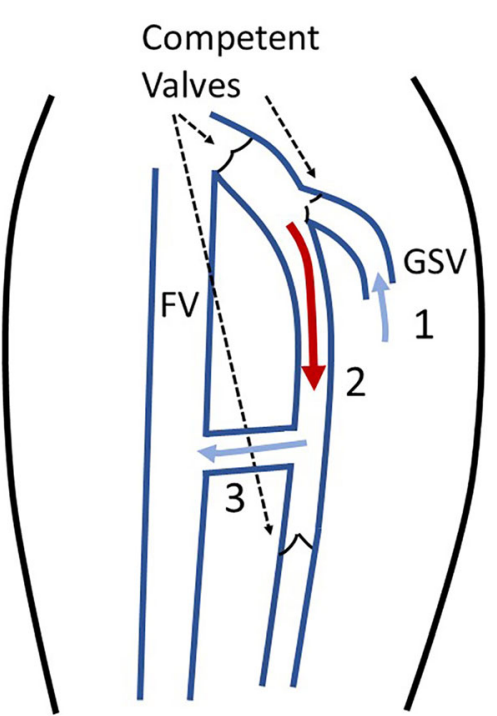

Fig. 1 Example of a segmental reflux without valvular incompetence. (1) Normally directed flow from a tributary into the great saphenous vein (GSV). (2) Reversed flow (reflux) in a segment of the GSV. (3) Normally directed flow from the GSV via a competent thigh perforating vein into the femoral vein $(\mathrm{FV})$. Note that all valves are competent 


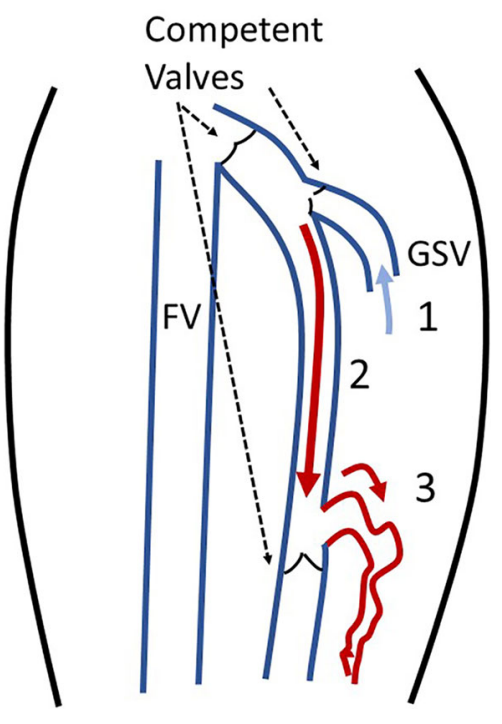

Fig. 2 Example of a segmental reflux without valvular incompetence in the GSV, but with varicose tributaries of the GSV. (1) Normally directed flow from a tributary into the great saphenous vein (GSV). (2) Reversed flow (reflux) in a segment of the GSV. (3) Reversed flow (reflux) in a varicose tributary of the GSV. Note that all valves in the GSV are competent more distal perforated vein (Fig. 1). This GSV segment will have a reversal of the flow (reflux) although all valves in the GSV, a tributary, and a perforator are normal and competent. More frequently, segmental reflux in the GSV occurs when the flow enters the GSV from its tributary and exits into a varicose vein connected distally (Fig. 2). A segmental reflux therefore does not always mean that the saphenous vein is abnormal and needs any treatment.

Axial reflux, on the other hand, always indicates the presence of incompetent valves. It occurs most frequently in the superficial system and can involve several veins. In addition to the axial GSV reflux (Fig. 3a), it can continue from the GSV via an intersaphenous vein into the SSV (Fig. 3b) or to an accessory saphenous vein (Fig. 3c). Hemodynamically, these and other variants of the anatomical extent of reflux are the same-the reversal of the flow is continuous from the groin to the calf, which is the definition of "axial reflux." If such terminology were to replace that describing specific venous segments, many problems with insurance policies (for example, related to treatment of accessory veins) might be resolved.
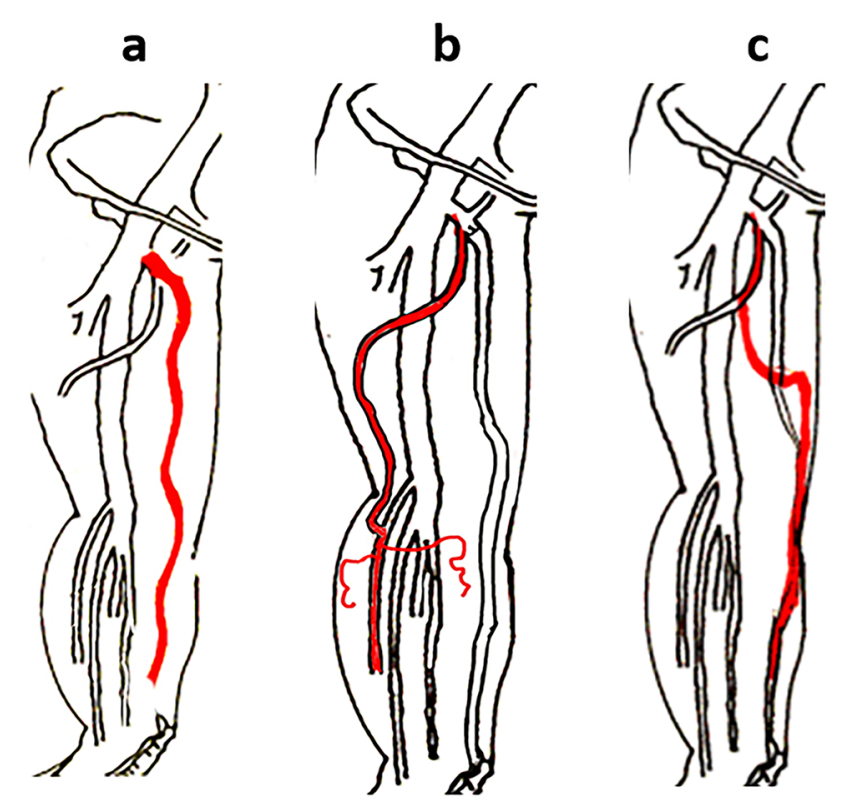

Fig. 3 Common variants of superficial axial reflux. a Axial GSV reflux; b axial reflux from the GSV via an and its varicose tributaries; $\mathbf{c}$ axial reflux from the accessory saphenous vein into distal segments of the GSV 
Standardizing definitions of axial, segmental, and multi-segmental reflux will lead to an accurate reflection of the anatomical extent of reflux in each extremity. Not only is this instrumental for scientific and clinical communication, but it would also help physicians make better clinical decisions and may improve communication with payors. This article is based on previously conducted studies and does not contain any studies with human participants or animals performed by any of the authors.

\section{ACKNOWLEDGEMENTS}

Funding. No funding or sponsorship was received for this study or publication of this article.

Authorship. All named authors meet the International Committee of Medical Journal Editors (ICMJE) criteria for authorship for this article, take responsibility for the integrity of the work as a whole, and have given their approval for this version to be published.

Disclosures. Fedor Lurie has no personal, financial, commercial, or academic conflicts of interest to disclose.

Compliance with Ethics Guidelines. This article is based on previously conducted studies and does not contain any studies with human participants or animals performed by any of the authors.

Data Availability. Data sharing is not applicable to this article as no datasets were generated or analyzed during the current study.

Open Access. This article is licensed under a Creative Commons Attribution-
NonCommercial 4.0 International License, which permits any non-commercial use, sharing, adaptation, distribution and reproduction in any medium or format, as long as you give appropriate credit to the original author(s) and the source, provide a link to the Creative Commons licence, and indicate if changes were made. The images or other third party material in this article are included in the article's Creative Commons licence, unless indicated otherwise in a credit line to the material. If material is not included in the article's Creative Commons licence and your intended use is not permitted by statutory regulation or exceeds the permitted use, you will need to obtain permission directly from the copyright holder. To view a copy of this licence, visit http:// creativecommons.org/licenses/by-nc/4.0/.

\section{REFERENCES}

1. Bernardini E, De Rango P, Piccioli R, et al. Development of primary superficial venous insufficiency: the ascending theory. Observational and hemodynamic data from a 9-year experience. Ann Vasc Surg. 2010;24(6):709-20.

2. Labropoulos N, Giannoukas AD, Delis K, et al. Where does venous reflux start? J Vasc Surg. 1997;26(5): $736-42$.

3. Engelhorn CA, Cassou MF, Engelhorn AL, SallesCunha SX. Does the number of pregnancies affect patterns of great saphenous vein reflux in women with varicose veins? Phlebology. 2010;25(4):190-5.

4. Coelho Neto F, de Oliveira RG, Gregório EP, Belczak SQ, de Araujo WJB. Saphenous reflux patterns in C2 patients: a record of 1196 ultrasound reports. Phlebology. 2019. https://doi.org/10.1177/ 0268355519889868 .

5. Perrin M, Eklof B, Maleti O. The vein glossary. J Vasc Surg Venous Lymphat Disord. 2018;5(6):e11-e217. 